\title{
Properties and Applications of the Eigenvector Corresponding to the Laplacian Spectral Radius of a Graph
}

\author{
Haizhou Song and Qiufen Wang \\ College of Mathematical Sciences, Huaqiao University, Quanzhou, Fujian 362021, China \\ Correspondence should be addressed to Haizhou Song; hzsong@hqu.edu.cn
}

Received 26 November 2012; Revised 26 February 2013; Accepted 26 February 2013

Academic Editor: Yong-Kui Chang

Copyright (C) 2013 H. Song and Q. Wang. This is an open access article distributed under the Creative Commons Attribution License, which permits unrestricted use, distribution, and reproduction in any medium, provided the original work is properly cited.

We study the properties of the eigenvector corresponding to the Laplacian spectral radius of a graph and show some applications. We obtain some results on the Laplacian spectral radius of a graph by grafting and adding edges. We also determine the structure of the maximal Laplacian spectrum tree among trees with $n$ vertices and $k$ pendant vertices $(n, k$ fixed), and the upper bound of the Laplacian spectral radius of some trees.

\section{Introduction}

The theory of graph spectra has been established in the 1950 s and 1960s. Chung has taken the investigation of the theory of graph spectra to a new level by a 45 -minute report [1] presented at the World Congress of Mathematicians in 1994, and in monographs [2]. Applications of the theory of graph spectra have also been found in the fields of electrical networks and vibration theory $[3,4]$. The wide range of the applications of the theory of graph spectra has led it to become a very active field of research of graph theory over the last thirty to forty years, and large numbers of results are continuously emerging.

There are many results on the (adjacency) spectral radius for different classes of graphs. Guo et al. [5] have studied the largest and the second largest spectral radius of trees with $n$ vertices and diameter $d$. Guo and Shao [6] have studied the first $\lfloor d / 2\rfloor+1$ (where $\lfloor x\rfloor$ represents the maximal integer not more than $x$ ) spectral radii of graphs with $n$ vertices and diameter $d$. Wu et al. [7] have studied the spectral radius of trees on $k$ pendant vertices.

There are also many results on the Laplacian spectral radius for different classes of graphs. Hong and Zhang [8] have studied the upper and lower bounds for the Laplacian spectral radius of trees. Guo [9] has studied the second largest Laplacian eigenvalues of trees. In the paper, we further study the properties of the eigenvector corresponding to the Laplacian spectral radius of a graph and obtain some applications on the Laplacian spectral radius of trees with $k$ pendant vertices.

Let $G=(V(G), E(G))$ be a simple connected graph with vertex set $V(G)=\left\{v_{1}, v_{2}, \ldots, v_{n}\right\}$ and edge set $E(G)$. Let $d_{G}\left(v_{i}\right)$ denote the degree of vertex $v_{i}(1 \leq i \leq n)$ of graph $G$ sometimes for convenience we denote by $d\left(v_{i}\right)$. If $D(G)=\operatorname{diag}\left(d\left(v_{1}\right), d\left(v_{2}\right), \ldots, d\left(v_{n}\right)\right)$ is the diagonal matrix of the vertex degree and $A(G)$ is the $(0,1)$ adjacency matrix of $G$, then the matrix $L(G)=D(G)-A(G)$ is called the Laplacian matrix of graph $G$. When $G$ is connected, $A(G)$ is irreducible, and, by the Perron-Frobenius theorem [10-12], the spectral radius $\rho(G)$ is simple and there is a unique positive unit eigenvector. We refer to such an eigenvector as the Perron vector of $G$. It is easy to see that $L(G)$ is a positive semidefinite $M$ matrix and its rows sum to $0, L(G)$ is singular. Hence, the eigenvalues of $L(G)$ can be ordered as $\mu_{1}(G) \geq \mu_{2}(G) \geq \cdots \geq$ $\mu_{n}(G)=0$. The largest eigenvalue $\mu_{1}(G)$ of $L(G)$ is called the Laplacian spectral radius of the graph $G$, denoted by $\mu(G)$. This has been used extensively in fields such as theoretical chemistry, combinatorial optimization, and communication networks $[13,14]$.

A pendant vertex of $G$ is a vertex of degree 1. Let $N_{G}(v)$ denote the adjacent vertex set of $v$ in $G$ and $d_{v}$ the degree of $v$. $\mathscr{T}_{n, k}$ denotes the set of trees for $n$ vertices and $k$ pendant vertices ( $n, k$ fixed). The tree $T_{n, k}$ is obtained from a star $K_{1, k}$ 


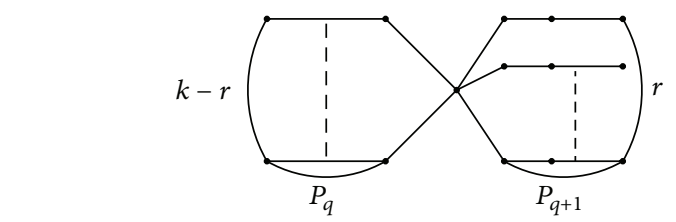

FIGURE 1

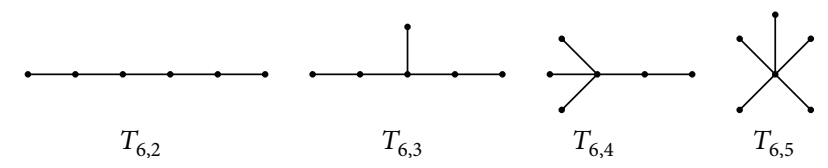

Figure 2

and $k$ paths of almost equal length by joining each pendant vertex of $K_{1, k}$ to an end of one path. Obviously, $2 \leq k \leq n-1$. Assume $q=[(n-1) / k], n-1=k q+r(0 \leq r \leq k-1) ; T_{n, k}$ is shown in Figure 1.

In general, $T_{n, n-1}=K_{1, n-1}, T_{n, 2}=P_{n}$, and $T_{n, n-2}=S(1, n-$ 3 ), a tree is obtained from two stars $K_{1, n-3}$ and $K_{1,1}$ by joining their central vertices. For example, $T_{6,5}=K_{1,5}, T_{6,4}=S(1,3)$, and $T_{6,2}$ is a path of length 6 (Figure 2).

In Section 2, we describe some properties of the eigenvector corresponding to the Laplacian spectral radius of a graph. Section 3 presents some applications of the eigenvector corresponding to the Laplacian spectral radius of a graph, including some results on the Laplacian spectral radius of a graph by grafting and adding edges. Then we obtain the structure of the maximal Laplacian spectrum tree among trees with $n$ vertices and $k$ pendant vertices ( $n, k$ fixed), and the upper bound of the Laplacian spectral radius for some trees.

\section{Properties of the Eigenvector Corresponding to the Laplacian Spectral Radius of a Graph}

For convenience, we denote $L(G)$ by $L$ sometimes. Since the Laplacian matrix $L(G)$ is a real symmetric matrix, we have the following theorem.

Theorem 1. Let $G$ be a simple connected graph of $n$ vertices. $L(G)$ is the Laplacian matrix of $G$, with vector $x \in \mathscr{R}^{n \times 1}$. Then

(1) $\mu(G)=\max _{1 \leq i \leq n} \mu_{i}$, where $\mu_{i}$ is the eigenvalue of $L(G)$;

(2) $\mu(G)=\max _{\|x\|=1}\left(x^{\prime} L x\right)$;

(3) if $\|x\|=1$ and $x^{\prime} L(G) x=\mu(G)$, then $L(G) x=\mu(G) x$.

Proof. (1) It is proved by the definition.

(2) Since $L(G)$ is a real symmetric matrix, there exists an orthogonal matrix $P$ for which $P^{-1} L P=$ $\operatorname{diag}\left(\mu_{1}, \mu_{2}, \ldots, \mu_{n}\right) \triangleq D$, where $P=\left(W_{1}, W_{2}, \ldots, W_{n}\right)$. Let $x=P y$, so $x^{\prime} L x=y^{\prime} P^{\prime} L P y=y^{\prime} P^{-1} L P y=y^{\prime} D y=$ $\sum_{i=1}^{n} \mu_{i} y_{i}^{2}$
$P$ is an orthogonal matrix and $x=P y$. Then, when $\|x\|=$ 1 , we have $\|y\|=1$. Assume, without loss of generality, that $\mu_{1} \leq \mu_{2} \leq \cdots \leq \mu_{n}$. Thus,

$$
\begin{aligned}
\max _{\|x\|=1}\left(x^{\prime} L x\right) & =\max _{\|y\|=1} \sum_{i=1}^{n} \mu_{i} y_{i}^{2} \leq \max _{\|y\|=1} \sum_{i=1}^{n} \mu_{n} y_{i}^{2} \\
& =\mu_{n} \max _{\|y\|=1} \sum_{i=1}^{n} y_{i}^{2}=\mu_{n}=\mu(G) .
\end{aligned}
$$

Let $y=e_{n}=(0,0, \ldots, 0,1)_{n \times 1}^{\prime}$, so we have $x=P y=$ $W_{n}$, and then the above equality holds. Therefore, $\mu(G)=$ $\max _{\|x\|=1}\left(x^{\prime} L x\right)$.

(3) Since $L(G)$ is a real symmetric matrix, there exists an orthogonal matrix $P$ for which $P^{-1} L P=$ $\operatorname{diag}\left(\mu_{1}, \mu_{2}, \ldots, \mu_{n}\right) \triangleq D$, where $P=\left(W_{1}, W_{2}, \ldots, W_{n}\right)$, and $W_{i}$ is the eigenvector corresponding to $\mu_{i}$ of $L(G)$. Assume, without loss of generality, that $\mu_{1} \leq \mu_{2} \leq \cdots \leq \mu_{n}$.

Let $x=P y$, and then $x^{\prime} L(G) x=y^{\prime} P^{\prime} L P y=y^{\prime} D y$. Since $x^{\prime} L(G) x=\mu(G)$, we have $x^{\prime} L(G) x=y^{\prime} D y=\mu_{n}=\mu(G)$. Thus, $y=e_{n}=(0,0, \ldots, 0,1)_{n \times 1}^{\prime}$ and then $x=P y=P e_{n}=$ $W_{n}$. However, $W_{n}$ is the eigenvector corresponding to $\mu_{n}$ of $L(G)$, that is, $L(G) W_{n}=\mu_{n} W_{n}$. Thus, $L(G) x=\mu(G) x$.

Definition 2. Let $G$ be a simple connected graph, $G=(V, E)$. If vector $x \in \mathscr{R}^{n \times 1}$ satisfies $\|x\|=1$ and $x^{\prime} L(G) x=\mu(G)$, then we call $x$ the standard Laplacian spectral vector of $G$.

Theorem 3. Let $T$ be a tree, and $V(T)=\left\{v_{1}, v_{2}, \ldots, v_{n}\right\}$. Let $L$ be the Laplacian matrix of $T$ and $x$ be the standard Laplacian spectral vector of $T$. Then

(1) $x$ is a real vector;

(2) $|x|>0$.

Proof. (1) Since $L$ is a real symmetric matrix, we have $\mu(T) \epsilon$ $R$. Thus, $x$ is a real vector.

(2) Suppose that $|x|>0$ is not established. Then there exists vertex set $H=\left\{v_{j_{1}}, v_{j_{2}}, \ldots, v_{j_{t}}\right\}$, which means $x_{j_{i}}=$ $0(i=1,2, \ldots, t)$, where $x_{j}$ corresponds to vertex $v_{j}$. Since $x$ is the standard Laplacian spectral vector of $T$, we have $\|x\|=1$. Thus, $x \neq 0$ and there exists vertex $v \in V(T)$ that makes $x_{v}=0$ and vertex $u \in N_{T}(v)$ that makes $x_{u} \neq 0$.

Let $T$ form a root tree with $v$ as its root vertex. $N_{T}(v)=$ $\left\{w_{1}, w_{2}, \ldots, w_{s}\right\} \triangleq W$. Let $T_{i}$ be the subtree of $T$ obtained from vertex $w_{i}$ and all its descendants. Let $y_{v}=x_{v}=0, y_{w_{i}}=$ $\left|x_{w_{i}}\right|(i=1,2, \ldots, s), y_{j}=x_{j}$ when $x_{w_{i}} \geq 0$, and let $v_{j}$ be the vertex of subtree $T_{i}, y_{j}=-x_{j}$ when $x_{w_{i}}<0$ and $v_{j}$ be the vertex of subtree $T_{i}(i=1,2, \ldots, s)$.

Thus, we have $y_{w_{i}} \geq 0(i=1,2, \ldots, s),\|y\|=1$ and $y^{\prime} L y=$ $x^{\prime} L x=\mu(L(T)) \triangleq \mu(T)$. By Definition 2, we know that $y$ is the standard Laplacian spectral vector of $T$. Then $(D-A) y=$ $\mu(T) y$. We have $((D-A) y)_{v}=(\mu(T) y)_{v}$. Thus, $\sum_{i=1}^{s} y_{w_{i}}=0$.

However, $y_{w_{i}} \geq 0$ and there exists $i_{0} \in\{1,2, \ldots, s\}$ that makes $w_{i_{0}}=u, y_{u}>0$. Therefore, $\sum_{i=1}^{s} y_{w_{i}}>0$. This contradiction completes the proof.

Theorem 4. Let $v$ be a vertex of tree $T$ and $v_{1}, v_{2}, \ldots, v_{s}$ be the pendant vertices of $T$ that are adjacent to $v$. If $x=$ 


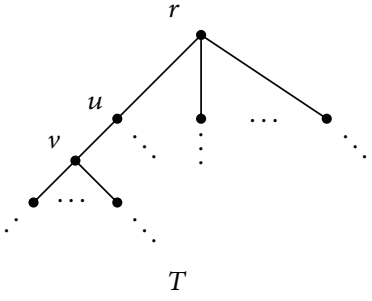

FIGURE 3

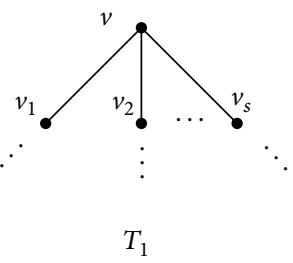

FIGURE 4

$\left(x_{1}, x_{2}, \ldots, x_{n}\right)^{\prime}$ is the standard Laplacian spectral vector of $T$, where $x_{i}$ corresponds to the vertex $v_{i}(1 \leq i \leq n)$. Then $x_{v_{j}}=x_{v_{i}}, 1 \leq i<j \leq s$.

Proof. $x=\left(x_{1}, x_{2}, \ldots, x_{n}\right)^{\prime}$ is the standard Laplacian spectral vector of $T$. By Definition 2 and Theorem 1, we have $L(T) x=$ $\mu(T) x . L(T)=D(T)-A(T)$, so $(D(T)-A(T)) x=\mu(T) x$, and then we have $\left(d_{T}\left(v_{i}\right)-\mu(T)\right) x_{v_{i}}=x_{v}, 1 \leq i \leq s$. Therefore, we have $(1-\mu(T)) x_{v_{1}}=x_{v},(1-\mu(T)) x_{v_{2}}=x_{v}, \ldots,(1-\mu(T)) x_{v_{s}}=$ $x_{v}$. Thus, $x_{v_{j}}=x_{v_{i}}, 1 \leq i<j \leq s$.

Theorem 5. Let $x=\left(x_{1}, x_{2}, \ldots, x_{n}\right)^{\prime}$ be the standard Laplacian spectral vector of tree $T$, where $x_{i}$ corresponds to vertex $v_{i}(1 \leq i \leq n), T=(V, E)$. Then

(1) for $\forall u v \in E(T)$, we have $x_{u} x_{v}<0$;

(2) $\sum_{i=1}^{n} x_{i}=0$.

Proof. (1) Since $x$ is the standard Laplacian spectral vector of $T$, by Definition 2 we have $\mu(T)=x^{\prime} L(T) x=\sum_{(i, j) \in E}\left(x_{i}-\right.$ $\left.x_{j}\right)^{2}$. By Theorem 3, we have $\left|x_{u}\right|>0$ and $\left|x_{v}\right|>0$. Thus, $x_{u} x_{v} \neq 0$.

Suppose that there exists edge $u v \in E(T)$ such that $x_{u} x_{v}>$ 0 . Let tree $T$ form a root tree with $r$ as its root vertex. Assume, without loss of generality, that vertex $u$ is the parent node of the vertex $v$ (Figure 3 ). Let $T_{1}$ be the subtree of $T$ obtained from vertex $v$ and all its descendants, and let $T_{1}$ be a $k$-layer tree, $T_{1}=\left(V_{1}, E_{1}\right)$ (Figure 4$)$. Without loss of generality, we assume that $x_{u}>0$, so $x_{v}>0$.

Let $y_{i}=x_{i}\left(v_{i} \in V \backslash V\left(T_{1}\right)\right), y_{v}=-x_{v}$, and $y_{w_{1}}=\left|x_{w_{1}}\right|\left(\forall w_{1}\right.$ are the vertices in the second layer of $\left.T_{1}\right)$, $y_{w_{2}}=(-1)^{3}\left|x_{w_{2}}\right|\left(\forall w_{2}\right.$ are the vertices in the third layer of
$\left.T_{1}\right), \ldots, y_{w_{k-1}}=(-1)^{k}\left|x_{w_{k-1}}\right|\left(\forall w_{k-1}\right.$ are the vertices in the $k$ th layer of $\left.T_{1}\right)$. Then $\|y\|=\|x\|=1$ and we have

$$
\begin{aligned}
y^{\prime} L & (T) y \\
& =\sum_{(i, j) \in E}\left(y_{i}-y_{j}\right)^{2}
\end{aligned}
$$

$$
\begin{aligned}
& =\sum_{(i, j) \in E \backslash E_{1}}\left(x_{i}-x_{j}\right)^{2}+\sum_{(i, j) \in E_{1} \backslash u v}\left(y_{i}-y_{j}\right)^{2}+\left(x_{u}+x_{v}\right)^{2} \\
& >\sum_{(i, j) \in E \backslash E_{1}}\left(x_{i}-x_{j}\right)^{2}+\sum_{(i, j) \in E_{1} \backslash u v}\left(x_{i}-x_{j}\right)^{2}+\left(x_{u}-x_{v}\right)^{2} \\
& =\sum_{(i, j) \in E}\left(x_{i}-x_{j}\right)^{2}=\mu(T) .
\end{aligned}
$$

Thus, there exists a unit vector $y=\left(y_{1}, y_{2}, \ldots, y_{n}\right)^{\prime}$ such that $\mu(T)<y^{\prime} L(T) y$. This implies a contradiction with Theorem 1. Therefore, $x_{u} x_{v}<0$.

(2) Since $x$ is the standard Laplacian spectral vector of $T$, we have

$$
(D(T)-A(T)) x=\mu(T) x .
$$

Then

$$
\left(d_{T}\left(v_{i}\right)-\mu(T)\right) x_{i}=\sum_{v_{j} \in N_{T}\left(v_{i}\right)} x_{j} .
$$

Thus, we have

$$
\sum_{i=1}^{n}\left(d_{T}\left(v_{i}\right)-\mu(T)\right) x_{i}=\sum_{i=1}^{n} \sum_{v_{j} \in N_{T}\left(v_{i}\right)} x_{j}
$$

Therefore, $\sum_{i=1}^{n} d_{T}\left(v_{i}\right) x_{i}-\mu(T) \sum_{i=1}^{n} x_{i}=\sum_{i=1}^{n} d_{T}\left(v_{i}\right) x_{i}$. So $\sum_{i=1}^{n} x_{i}=0$.

\section{Applications of the Eigenvector Corresponding to the Laplacian Spectral Radius}

In this section, we present some applications of the eigenvector of the Laplacian spectral radius of a graph.

Theorem 6. Let $u$ and $v$ be the vertices of tree T. Denote the distance of vertices $u$ and $v$ by $d(u, v)$ and $d(u, v)=k$, where $k$ is an odd number and $k \geq 3$. Let $G^{\prime}$ be the graph obtained from $T$ by adding edge $(u, v)$. Then $\mu\left(G^{\prime}\right)>\mu(T)$.

Proof. Let $x=\left(x_{1}, x_{2}, \ldots, x_{n}\right)^{\prime}$ be the standard Laplacian spectral vector of tree $T$, where $x_{i}$ corresponds to vertex 
$v_{i}(1 \leq i \leq n)$. Since $k$ is an odd number and $k \geq 3$, by Theorem 5 we have $x_{u} x_{v}<0$. Thus,

$$
\begin{aligned}
\mu\left(G^{\prime}\right) & =\max _{\|y\|=1} y^{\prime} L\left(G^{\prime}\right) y \\
& =\max _{\|y\|=1} \sum_{(i, j) \in E\left(G^{\prime}\right)}\left(y_{i}-y_{j}\right)^{2} \\
& \geq \sum_{(i, j) \in E\left(G^{\prime}\right)}\left(x_{i}-x_{j}\right)^{2} \\
& >\sum_{(i, j) \in E(T)}\left(x_{i}-x_{j}\right)^{2}=\mu(T) .
\end{aligned}
$$

Therefore, $\mu\left(G^{\prime}\right)>\mu(T)$.

According to the well-known Courant-Weyl inequalities, we have the following lemma.

Lemma 7 (see [15]). Let $G$ be a graph with $n$ vertices and let $G^{\prime}=G+e$ be the graph obtained by inserting a new edge e into $G$. Let $\mu_{i}(G)$ and $\mu_{i}\left(G^{\prime}\right)$ be the eigenvalues of the Laplacian matrix of $G$ and $G^{\prime}(i=1,2, \ldots, n)$, respectively. Then $\mu_{1}\left(G^{\prime}\right) \geq \mu_{1}(G) \geq \mu_{2}\left(G^{\prime}\right) \geq \mu_{2}(G) \geq \cdots \geq \mu_{n}\left(G^{\prime}\right)=$ $\mu_{n}(G)=0$.

Lemma 8 (see [16]). Let $G$ be a connected graph for $n$ vertices with at least one edge. Then $\mu(G) \geq \Delta(G)+1$, where $\Delta(G)$ is the largest degree of the graph $G$, and equality holds if and only if $\Delta(G)=n-1$.

Theorem 9. Let $G$ be a connected graph for $n$ vertices, and let $u_{j}$ be the vertex of $G$ and $v_{1}^{j}, v_{2}^{j}, \ldots, v_{m_{j}}^{j}$ be the pendant vertices of $G$ adjacent to vertex $u_{j}(1 \leq j \leq k)$. Let $G_{1}$ be the graph obtained from $G$ by adding $\sum_{j=1}^{k} q_{j}\left(1 \leq q_{j} \leq m_{j}\left(m_{j}-\right.\right.$ $1) / 2)$ edges, where $t_{j}$ edges are among $v_{1}^{j}, v_{2}^{j}, \ldots, v_{m_{j}}^{j}(j=$ $1,2, \ldots, k)$. Then $\mu(G)=\mu\left(G_{1}\right)$.

Proof. If $\Delta(G)=n-1$, by Lemma 8 we have $\mu(G)=\Delta(G)+1=$ $n$. However, it is clear that $\mu\left(G_{1}\right)=\Delta\left(G_{1}\right)+1=n-1+1=n$, so $\mu(G)=\mu\left(G_{1}\right)$. We suppose that $\Delta(G) \leq n-2$.

Let $G^{\prime}$ be the graph obtained from $G$ by adding $\sum_{j=1}^{k} t_{j}\left(t_{j}=m_{j}\left(m_{j}-1\right) / 2\right)$ edges, where $t_{j}$ edges are among $v_{1}^{j}, v_{2}^{j}, \ldots, v_{m_{j}}^{j}(j=1,2, \ldots, k)$. By Lemma 7 , we have $\mu(G) \leq$ $\mu\left(G_{1}\right) \leq \mu\left(G^{\prime}\right)$. To obtain the result, we only need to prove that $\mu(G) \geq \mu\left(G^{\prime}\right)$.

Let $X=\left(X_{1}, X_{2}, \ldots, X_{n}\right)^{\prime}$ be the standard Laplacian spectral vector of $G^{\prime}$, then $L\left(G^{\prime}\right) X=\mu\left(G^{\prime}\right) X$. For $L\left(G^{\prime}\right)=$ $D\left(G^{\prime}\right)-A\left(G^{\prime}\right)$, we have

$$
\begin{array}{r}
\left(d_{G^{\prime}}\left(v_{i}^{l}\right)-\mu\left(G^{\prime}\right)\right) X_{v_{i}^{l}}=X_{u_{l}}+\sum_{j=1, j \neq i}^{m_{l}} X_{v_{j}^{l}}, \\
1 \leq i \leq m_{l}, \quad 1 \leq l \leq k .
\end{array}
$$
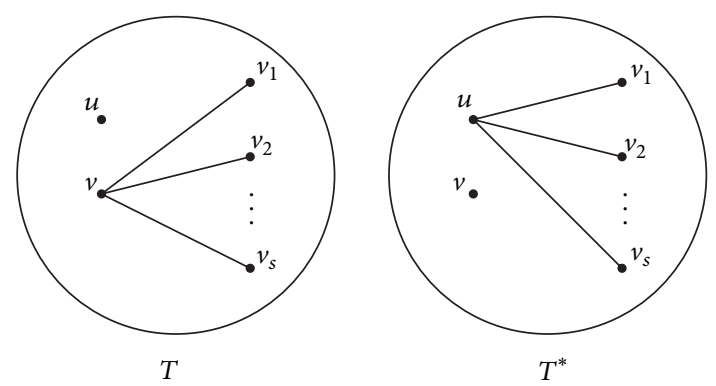

FIGURE 5

Then, for $1 \leq i<j \leq m_{l}$ and $1 \leq l \leq k$, we have

$$
\begin{aligned}
& \left(d_{G^{\prime}}\left(v_{i}^{l}\right)-\mu\left(G^{\prime}\right)\right) X_{v_{i}^{l}}-\left(d_{G^{\prime}}\left(v_{j}^{l}\right)-\mu\left(G^{\prime}\right)\right) X_{v_{j}^{l}} \\
& \quad=X_{v_{j}^{l}}-X_{v_{i}^{l}}
\end{aligned}
$$

since $d_{G^{\prime}}\left(v_{1}^{l}\right)=d_{G^{\prime}}\left(v_{2}^{l}\right)=\cdots=d_{G^{\prime}}\left(v_{q_{l}}^{l}\right)=m_{l} \leq n-2$. Thus,

$$
\begin{array}{r}
\left(m_{l}-\mu\left(G^{\prime}\right)\right)\left(X_{v_{i}^{l}}-X_{v_{j}^{l}}\right)=X_{v_{j}^{l}}-X_{v_{i}^{l}}, \\
1 \leq i<j \leq m_{l}, \quad 1 \leq l \leq k .
\end{array}
$$

For $\Delta\left(G^{\prime}\right) \leq n-2$, by Lemma 8 we have $\mu\left(G^{\prime}\right)>\Delta\left(G^{\prime}\right)+$ $1 \geq m_{l}+1$. So $X_{v_{i}^{l}}=X_{v_{j}^{l}}, 1 \leq i<j \leq m_{l}, 1 \leq l \leq k$. Thus, we have

$$
\begin{aligned}
\mu(G) & \geq X^{\prime} L(G) X=\sum_{(i, j) \in E(G)}\left(X_{i}-X_{j}\right)^{2} \\
& =\sum_{(i, j) \in E\left(G^{\prime}\right)}\left(X_{i}-X_{j}\right)^{2} \\
& =X^{\prime} L\left(G^{\prime}\right) X=\mu\left(G^{\prime}\right) .
\end{aligned}
$$

That is, $\mu(G) \geq \mu\left(G^{\prime}\right)$. This completes the proof.

Theorem 10. Let $u, v$ be two vertices of tree T. Suppose that $v_{1}, v_{2}, \ldots, v_{s}\left(1 \leq s \leq d_{v}\right)$ are some vertices of $N_{T}(v) \backslash\left(N_{T}(u) \cup\right.$ $\{u\})$, and $x=\left(x_{1}, x_{2}, \ldots, x_{n}\right)^{\prime}$ is the standard Laplacian spectral vector of $T$, where $x_{i}$ corresponds to vertex $v_{i}(1 \leq i \leq$ $n)$. Let $T^{*}$ be the tree obtained from $T$ by deleting the edges $\left(v, v_{i}\right)$ and adding the edges $\left(u, v_{i}\right)(1 \leq i \leq s)$ (Figure 5). If $\left|x_{u}\right| \geq\left|x_{v}\right|$, then $\mu(T)<\mu\left(T^{*}\right)$.

Proof. For tree $T$, let $T$ form a root tree with vertex $v$ as the parent node of vertex $v_{i}(1 \leq i \leq s), T=(V, E)$. Let $T_{1}$ be the subtree of $T$ obtained from the parent node $v$ and $v_{1}, v_{2}, \ldots, v_{s}$ and the $v_{1}$ descendants, $v_{2}$ descendants,.., $v_{s}$ descendants, $T_{1}=\left(V_{1}, E_{1}\right)$. For tree $T^{*}$, let $T^{*}$ form a root tree with vertex $u$ as the parent node of vertex $v_{i}(1 \leq i \leq s)$, $T^{*}=\left(V^{*}, E^{*}\right)$. Let $T_{1}^{*}$ be the subtree of $T^{*}$ obtained from the parent node $u$ and $v_{1}, v_{2}, \ldots, v_{s}$ and the $v_{1}$ descendants, $v_{2}$ descendants,..., and $v_{s}$ descendants. Let subtree $T_{1}^{*}$ be a $k$-layer tree with vertex $u$ as the root node. Consider $E_{1}^{\prime}=$ $E_{1} \backslash\left\{v v_{1}, v v_{2}, \ldots, v v_{s}\right\}$. 
Let $y_{i}=x_{i}\left(v_{i} \in V \backslash V\left(T_{1}\right)\right), y_{v}=x_{v}, y_{w_{1}}=$ - $\left(\operatorname{sign} x_{u}\right)\left|x_{w_{1}}\right|$ (for all $w_{1}$ are the vertices in the second layer of $\left.T_{1}^{*}\right), y_{w_{2}}=(-1)^{2}\left(\operatorname{sign} x_{u}\right)\left|x_{w_{2}}\right|\left(\right.$ for all $w_{2}$ are the vertices in the third layer of $\left.T_{1}^{*}\right), \ldots$, and $y_{w_{k-1}}=(-1)^{k-1}\left(\operatorname{sign} x_{u}\right)\left|x_{w_{k-1}}\right|$ (for all $w_{k-1}$ are the vertices in the $k$ th layer of $T_{1}^{*}$ ). Then we have $\|y\|=1$ and

$$
\begin{aligned}
y^{\prime} L & \left(T^{*}\right) y-x^{\prime} L(T) x \\
= & \sum_{(i, j) \in E^{*}}\left(y_{i}-y_{j}\right)^{2}-\sum_{(i, j) \in E}\left(x_{i}-x_{j}\right)^{2} \\
= & {\left[\sum_{(i, j) \in E_{1}^{\prime}}\left(y_{i}-y_{j}\right)^{2}+\sum_{(i, j) \in E^{*} \backslash E_{1}^{\prime}}\left(y_{i}-y_{j}\right)^{2}\right] } \\
& -\left[\sum_{(i, j) \in E_{1}^{\prime}}\left(x_{i}-x_{j}\right)^{2}+\sum_{(i, j) \in E \backslash E_{1}^{\prime}}\left(x_{i}-x_{j}\right)^{2}\right] \\
= & \sum_{(i, j) \in E^{*} \backslash E_{1}^{\prime}}\left(y_{i}-y_{j}\right)^{2}-\sum_{(i, j) \in E \backslash E_{1}^{\prime}}\left(x_{i}-x_{j}\right)^{2} \\
= & \sum_{i=1}^{s}\left(\left|x_{u}\right|+\left|x_{v_{i}}\right|\right)^{2}-\sum_{i=1}^{s}\left(\left|x_{v}\right|+\left|x_{v_{i}}\right|\right)^{2} \\
= & \sum_{i=1}^{s}\left(\left|x_{u}\right|^{2}-\left|x_{v}\right|^{2}\right)+2 \sum_{i=1}^{s}\left|x_{v_{i}}\right|\left(\left|x_{u}\right|+\left|x_{v}\right|\right) \geq 0 .
\end{aligned}
$$

That is, $y^{\prime} L\left(T^{*}\right) y \geq x^{\prime} L(T) x$. Thus,

$$
\mu(T)=x^{\prime} L(T) x \leq y^{\prime} L\left(T^{*}\right) y \leq \max _{\|y\|=1} y^{\prime} L\left(T^{*}\right) y=\mu\left(T^{*}\right) .
$$

Therefore, $\mu(T) \leq \mu\left(T^{*}\right)$.

If $\mu(T)=\mu\left(T^{*}\right)$, then the equalities in (12) hold. Thus, $\mu(T)=y^{\prime} L\left(T^{*}\right) y=\mu\left(T^{*}\right)$. By Theorem 1, we have $L\left(T^{*}\right) y=$ $\mu\left(T^{*}\right) y$. Thus,

$$
\mu\left(T^{*}\right) y_{v}=\left(L\left(T^{*}\right) y\right)_{v}=d_{v^{*}} y_{v}-\sum_{v_{i} \in N_{T^{*}}(v)} y_{i},
$$

where $d_{v^{*}}$ is the degree of vertex $v$ of tree $T^{*}$.

In addition, from $L(T) x=\mu(T) x$ we have

$$
\begin{aligned}
\mu(T) x_{v} & =(L(T) x)_{v} \\
& =d_{v} x_{v}-\sum_{v_{i} \in N_{T}(v)} x_{i} \\
& =d_{v} y_{v}-\sum_{v_{i} \in N_{T^{*}}(v)} x_{i}-\sum_{i=1}^{s} x_{i},
\end{aligned}
$$

where $d_{v}$ is the degree of vertex $v$ of tree $T$.

By (13) and (14), we have

$$
\left(\mu\left(T^{*}\right)-\mu(T)\right) x_{v}=\sum_{i=1}^{s} x_{i}-s x_{v}+\sum_{v_{i} \in N_{T^{*}}(v)}\left(x_{i}-y_{i}\right) .
$$

Assume, without loss of generality, that $x_{v}>0$.

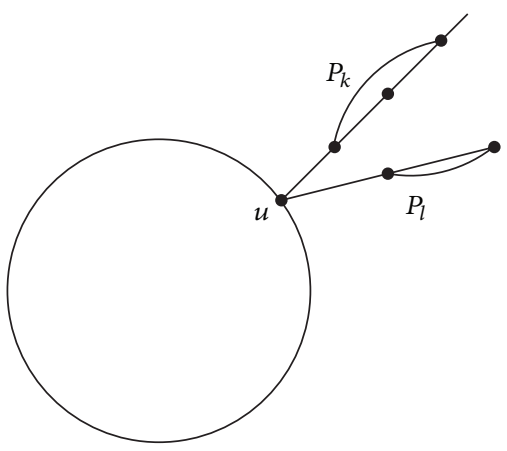

FigURE 6

If $x_{u}>0$, by Theorem 5 , we have

$$
\left(\mu\left(T^{*}\right)-\mu(T)\right) x_{v}=\sum_{i=1}^{s} x_{i}-s x_{v}<0
$$

which implies the contradiction $\mu\left(T^{*}\right)<\mu(T)$.

If $x_{u}<0$, by Theorem 5 , we have

$$
\left(\mu\left(T^{*}\right)-\mu(T)\right) x_{v}=\sum_{i=1}^{s} x_{i}-s x_{v}+\sum_{v_{i} \in N_{T^{*}}(v)}\left(x_{i}-\left|x_{i}\right|\right)<0,
$$

which implies the contradiction $\mu\left(T^{*}\right)<\mu(T)$.

Thus, if $\left|x_{u}\right| \geq\left|x_{v}\right|$, then $\mu(T)<\mu\left(T^{*}\right)$.

Corollary 11. Suppose that $T^{*}$ is as defined as in Theorem $10 y=\left(y_{1}, y_{2}, \ldots, y_{n}\right)^{T}$ is the standard Laplacian spectral vector of $T^{*}$, and then $\left|y_{u}\right|>\left|y_{v}\right|$.

Proof. Otherwise, if $\left|y_{u}\right| \leq\left|y_{v}\right|$, by Theorem 10, we have $\mu\left(T^{*}\right)<\mu(T)$. This contradiction completes the proof.

Corollary 12. Let $G$ be a simple connected graph with $n$ vertices. Let $r, s$, and $t$ be three distinct vertices, where $r s \in$ $E(G)$ and $r t \notin E(G)$. Let $G^{*}$ be the simple connected graph obtained from $G$ by deleting the edge $r$ s and adding the edge $r$. Suppose that $x=\left(x_{1}, x_{2}, \ldots, x_{n}\right)^{\prime}$ is the standard Laplacian spectral vector of $G$, where $x_{i}$ corresponds to the vertex $v_{i}(1 \leq$ $i \leq n) \cdot x^{*}=\left(x_{1}^{*}, x_{2}^{*}, \ldots, x_{n}^{*}\right)^{\prime}$ is the standard Laplacian spectral vector of $G^{*}$, where $x_{i}^{*}$ corresponds to the vertex $v_{i}^{*}(1 \leq i \leq n)$. If $\left|x_{t}\right| \geq\left|x_{s}\right|$, then $\left|x_{t}^{*}\right|>\left|x_{s}^{*}\right|$.

Proof. Obviously, $G$ is obtained from $G^{*}$ by deleting the edge $r t$ and adding the edge $r s$. Assume that $\left|x_{t}^{*}\right| \leq\left|x_{s}^{*}\right|$. Since $G^{*}$ is a simple connected graph, by Theorem 10 we have $\mu\left(G^{*}\right)<$ $\mu(G)$. If $\left|x_{t}\right| \geq\left|x_{s}\right|$, by Theorem 10 we have $\mu(G)<\mu\left(G^{*}\right)$. This contradiction completes the proof.

Lemma 13 (see [17]). Let $u$ be a vertex of tree $G$, and for positive integers $k$ and $l$ let $G_{k, l}$ denote the tree obtained from $G$ by adding pendant paths of length $k$ and $l$ at $u$ (Figure 6 ). If $k \geq l \geq 1$, then $\mu\left(G_{k, l}\right)>\mu\left(G_{k+1, l-1}\right)$. 
Theorem 14 (see $[7,8])$. Among all the trees for $n$ vertices and $k$ pendant vertices, the maximal Laplacian spectral radius is obtained uniquely at $T_{n, k}$.

Proof. We have to prove that if $T \in \mathscr{T}_{n, k}$, then $\mu(T) \leq \mu\left(T_{n, k}\right)$ with equality only when $T=T_{n, k}$. Let $t$ be the cardinality of the vertices of degree 3 or greater.

Case 1. $t=0$. In this case, $T$ is a path of length $n$, and hence $T=T_{n, 2}$. We have $\mu(T)=\mu\left(T_{n, 2}\right)$.

Case 2. $t=1$. The result $\mu(T) \leq \mu\left(T_{n, k}\right)$ follows from repeated use of Lemma 13, and equality holds if and only if $T=T_{n, k}$.

Case 3. $t>1$. Let $x=\left(x_{1}, x_{2}, \ldots, x_{n}\right)^{T}$ be the standard Laplacian spectral vector of $T$, where $x_{i}$ corresponds to the vertex $v_{i}(1 \leq i \leq n)$. Suppose that $u, v$ are two vertices of $T$ of degree 3 or greater, and $\left|x_{u}\right| \geq\left|x_{v}\right|$. Since $T$ is a tree, there is a unique path between $u$ and $v$, and only one of the neighbors of $v$, say $w$, is on the path. Assume $\left\{v_{1}, v_{2}, \ldots, v_{d_{v}-2}\right\} \subset N_{T}(v) \backslash\{w\}$. Delete the edges $\left(v, v_{i}\right)$ and add the edges $\left(u, v_{i}\right)\left(1 \leq i \leq d_{v}-2\right)$. Then we obtain a new tree $T_{1}^{*}$. Obviously, $T_{1}^{*}$ still has $k$ pendant vertices. By Theorem 10 , we have $\mu(T)<\mu\left(T_{1}^{*}\right)$ and the cardinality of the vertices of degree 3 or greater decreases to $t-1$.

If $t-1>1$, repeat the above step to $T_{1}^{*}$ until the cardinality is only 1 . Thus, we obtain trees $T_{2}^{*}, T_{3}^{*}, \ldots, T_{t-1}^{*}$ and $\mu\left(T_{1}^{*}\right)<$ $\mu\left(T_{2}^{*}\right)<\cdots<\mu\left(T_{t-1}^{*}\right)$. Moreover, each $T_{i}^{*}$ has $k$ pendant vertices. Referring to Case 2 , we have $\mu\left(T_{t-1}^{*}\right) \leq \mu\left(T_{n, k}\right)$. Therefore, $\mu(T)<\mu\left(T_{n, k}\right)$.

The cases complete the proof.

Theorem 15 (see [8]). $\mu\left(T_{n, k-1}\right)<\mu\left(T_{n, k}\right), 3 \leq k \leq n-1$.

Proof. The proof is obvious from Lemma 13.

For the sake of clarity, we identify a graph by its characteristic polynomial. Let $\Phi(L(G))$ be the characteristic polynomial of graph $G$. For convenience, we denote $\Phi(L(G))$ by $\Phi(G)$ sometimes. First, we require some lemmas.

Lemma 16 (see [9]). Let $e=u v$ be a cut edge of the simple connected graph G. $G_{1}$ and $G_{2}$ are two connected branches of $G-u v$, where $u \in V\left(G_{1}\right), v \in V\left(G_{2}\right)$. Then $\Phi(G)=$ $\Phi\left(G_{1}\right) \Phi\left(G_{2}\right)-\Phi\left(G_{1}\right) \Phi\left(L_{v}\left(G_{2}\right)\right)-\Phi\left(G_{2}\right) \Phi\left(L_{u}\left(G_{1}\right)\right)$. Here, $\Phi\left(L_{v}\left(G_{2}\right)\right)$ denotes the determinant obtained by deleting the row and column for vertex $v$ of $G_{2}$ from the determinant for $L\left(G_{2}\right)$, and $\Phi\left(L_{u}\left(G_{1}\right)\right)$ denotes the determinant obtained by deleting the row and column for vertex $u$ of $G_{1}$ from the determinant for $L\left(G_{1}\right)$.

Lemma 17 (see [18]). Let $P_{n}$ be a path with $n$ vertices, let $P_{n}^{0}$ be the path obtained by adding a loop on a pendant vertex in $P_{n}$, and let $P_{n}^{00}$ be the path obtained by adding loops on the two pendant vertices in $P_{n}$. Suppose that the degree of contribution corresponding to each loop is 1 and $\Phi\left(P_{0}\right)=0, \Phi\left(P_{0}^{0}\right)=1$, $\Phi\left(P_{0}^{00}\right)=1$. Then
(1) $\Phi\left(P_{n+1}\right)=(x-2) \Phi\left(P_{n}\right)+\Phi\left(P_{n-1}\right), \quad(n \geq 1)$,

(2) $x \Phi\left(P_{n}^{0}\right)=\Phi\left(P_{n+1}\right)+\Phi\left(P_{n}\right)$,

(3) $\Phi\left(P_{n}\right)=x \Phi\left(P_{n-1}^{00}\right),(n \geq 1)$,

(4) $\Phi\left(P_{n}\right)=\left(x / \sqrt{x^{2}-4 x}\right)\left(a^{n}-b^{n}\right)$, where $a=(x-2+$ $\left.\sqrt{x^{2}-4 x}\right) / 2$ and $b=\left(x-2-\sqrt{x^{2}-4 x}\right) / 2$.

From Lemma 16, by induction we can obtain the following lemma.

Lemma 18. Let $G_{1}, G_{2}, \ldots, G_{s}, G$ be $s+1$ disjoint simple connected graphs, where $v_{i} \in G_{i}(i=1,2, \ldots, s)$ and $v \in G$. Let $H_{s}$ be the graph obtained from $G_{1}, G_{2}, \ldots, G_{s}$ and $G$ by adding new edges $v v_{1}, v v_{2}, \ldots, v v_{s}$. Then

$$
\begin{aligned}
& \Phi\left(H_{s}\right) \\
& =\Phi(G) \prod_{i=1}^{s}\left[\Phi\left(G_{i}\right)-\Phi\left(L_{v_{i}}\left(G_{i}\right)\right)\right] \\
& \quad-\Phi\left(L_{v}(G)\right) \\
& \quad \times \sum_{i=1}^{s}\left[\Phi\left(G_{i}\right) \prod_{j=1, j \neq i}^{s}\left(\Phi\left(G_{j}\right)-\Phi\left(L_{v_{j}}\left(G_{j}\right)\right)\right)\right] .
\end{aligned}
$$

From Lemmas 16, 17, and 18, we have the following theorem.

Theorem 19. The characteristic polynomial of $T_{n, k}(2 \leq k \leq$ $n-1)$ is

$$
\begin{aligned}
\Phi\left(T_{n, k}\right)=\left[\Phi\left(P_{q+1}\right)-\Phi\left(P_{q}^{0}\right)\right]^{r-1}\left[\Phi\left(P_{q}\right)-\Phi\left(P_{q-1}^{0}\right)\right]^{k-r-1} \\
\times\left\{x\left[\Phi\left(P_{q+1}\right)-\Phi\left(P_{q}^{0}\right)\right]\left[\Phi\left(P_{q}\right)-\Phi\left(P_{q-1}^{0}\right)\right]\right. \\
-r\left[\Phi\left(P_{q}\right)-\Phi\left(P_{q-1}^{0}\right)\right] \Phi\left(P_{q+1}\right) \\
\left.-(k-r) \Phi\left(P_{q}\right)\left[\Phi\left(P_{q+1}\right)-\Phi\left(P_{q}^{0}\right)\right]\right\}
\end{aligned}
$$

where $q=[(n-1) / k], n-1=k q+r(0 \leq r \leq k-1)$, and $P_{q}$ is a path of length $q$.

As for the Laplacian spectral radius of $T_{n, k}$, we only need to consider the greatest root of

$$
\begin{aligned}
x & {\left[\Phi\left(P_{q+1}\right)-\Phi\left(P_{q}^{0}\right)\right]\left[\Phi\left(P_{q}\right)-\Phi\left(P_{q-1}^{0}\right)\right] } \\
& -r\left[\Phi\left(P_{q}\right)-\Phi\left(P_{q-1}^{0}\right)\right] \Phi\left(P_{q+1}\right) \\
& -(k-r) \Phi\left(P_{q}\right)\left[\Phi\left(P_{q+1}\right)-\Phi\left(P_{q}^{0}\right)\right]=0 .
\end{aligned}
$$

In general, the Laplacian spectral radius is difficult to calculate although the characteristic polynomial can be identified by Chebvshev polynomials. Thus, we only give some results in some cases. 
Lemma 20. For a univariate cubic equation $x^{3}+k x^{2}+m x+$ $n=0$, we can obtain the roots as follows:

$$
\begin{aligned}
x_{1}= & \sqrt[3]{-\frac{q}{2}+\sqrt{\left(\frac{q}{2}\right)^{2}+\left(\frac{p}{3}\right)^{3}}} \\
& +\sqrt[3]{-\frac{q}{2}-\sqrt{\left(\frac{q}{2}\right)^{2}+\left(\frac{p}{3}\right)^{3}}}-\frac{k}{3}, \\
x_{2}= & \omega \sqrt[3]{-\frac{q}{2}+\sqrt{\left(\frac{q}{2}\right)^{2}+\left(\frac{p}{3}\right)^{3}}} \\
& +\omega^{2} \sqrt[3]{-\frac{q}{2}-\sqrt{\left(\frac{q}{2}\right)^{2}+\left(\frac{p}{3}\right)^{3}}}-\frac{k}{3}, \\
x_{3}= & \omega^{2} \sqrt[3]{-\frac{q}{2}+\sqrt{\left(\frac{q}{2}\right)^{2}+\left(\frac{p}{3}\right)^{3}}} \\
& +\omega \sqrt[3]{-\frac{q}{2}-\sqrt{\left(\frac{q}{2}\right)^{2}+\left(\frac{p}{3}\right)^{3}}}-\frac{k}{3},
\end{aligned}
$$

where $p=-(1 / 3) k^{2}+m, q=(2 / 27) k^{3}-(1 / 3) k m+n$ and $\omega=e^{2 \pi i / 3}$.

Proof. The lemma is easy to prove.

Theorem 21. If $[n / 2] \leq k \leq n-1$, then

$$
\begin{array}{r}
\mu\left(T_{n, k}\right)=\frac{k+4}{3}+\frac{2 \sqrt{k^{2}-k+4}}{3} \cos \frac{\theta}{3}, \\
\theta=\arccos \frac{2 k^{3}-3 k^{2}-48 k+27 n-16}{2\left(k^{2}-k+4\right)^{3 / 2}}, \\
(0<\theta<\pi) .
\end{array}
$$

Moreover, if $k=n-1$, then $\mu\left(T_{n, n-1}\right)=n$.

Proof. In this case, for $[n / 2] \leq k \leq n-1, q=1$. By (20), we have

$$
\begin{aligned}
x[\Phi & \left.\left(P_{q+1}\right)-\Phi\left(P_{q}^{0}\right)\right]\left[\Phi\left(P_{q}\right)-\Phi\left(P_{q-1}^{0}\right)\right] \\
& -r\left[\Phi\left(P_{q}\right)-\Phi\left(P_{q-1}^{0}\right)\right] \Phi\left(P_{q+1}\right) \\
& -(k-r) \Phi\left(P_{q}\right)\left[\Phi\left(P_{q+1}\right)-\Phi\left(P_{q}^{0}\right)\right]=0 .
\end{aligned}
$$

Hence, $x^{3}-(k+4) x^{2}+(3 k+4) x-(k+r+1)=0$.
Let $f(x)=x^{3}-(k+4) x^{2}+(3 k+4) x-(k+r+1)$, and then we have

$1^{o}$ for $f(0)=-(k+r+1)<0$ and $f(1)=k-r>0$, and thus $f(x)$ has a root in $(0,1)$,

$2^{o}$ for $f(1)>0$ and $f(k)=-k(k-3)-r-1<0$, and thus $f(x)$ has a root in $(1, k)$,

$3^{o}$ for $f(k)<0$ and $f(+\infty)>0$, and thus $f(x)$ has a root in $(k,+\infty)$.

Therefore, the equation has three different real roots.

Let $x=y+(k+4) / 3$, and then the equation can translate to $y^{3}+p y+q=0$, where $p=-(1 / 3)\left(k^{2}-k+4\right)$ and $q=$ $-(1 / 27)\left(2 k^{3}-3 k^{2}-21 k+27 r+11\right)$.

Let $s=-q / 2+\sqrt{-\Delta} i=\|s\| e^{i \theta}$, where $\Delta=(q / 2)^{2}+(p / 3)^{3}<$ 0 . Then

$$
\begin{aligned}
\|s\| & =\left\|-\frac{q}{2}+\sqrt{-\Delta} i\right\| \\
& =\sqrt{\left(-\frac{q}{2}\right)^{2}+(-\Delta)}=\sqrt{\frac{q^{2}}{4}-\frac{q^{2}}{4}-\frac{p^{3}}{27}} \\
& =\frac{1}{27}\left(k^{2}-k+4\right)^{3 / 2},
\end{aligned}
$$

$\theta=\arccos \frac{-q / 2}{\|s\|}=\arccos \frac{2 k^{3}-3 k^{2}-21 k+27 r+11}{2\left(k^{2}-k+4\right)^{3 / 2}}$,

$$
(0<\theta<\pi) \text {. }
$$

Thus, based on the Lemma 20, we can obtain three roots:

$$
\begin{aligned}
& x_{1}=\sqrt[3]{-\frac{q}{2}+\sqrt{-\Delta}} i+\sqrt[3]{-\frac{q}{2}-\sqrt{-\Delta}} i+\frac{k+4}{3} \\
& =\sqrt[3]{\|s\| e^{i \theta}}+\sqrt[3]{\|s\| e^{-i \theta}}+\frac{k+4}{3} \\
& =\frac{\sqrt{k^{2}-k+4}}{3} e^{i \theta / 3}+\frac{\sqrt{k^{2}-k+4}}{3} e^{-i \theta / 3}+\frac{k+4}{3} \\
& =\frac{k+4}{3}+\frac{2 \sqrt{k^{2}-k+4}}{3} \cos \frac{\theta}{3}, \\
& x_{2}=\omega \sqrt[3]{-\frac{q}{2}+\sqrt{-\Delta}} i+\omega^{2} \sqrt[3]{-\frac{q}{2}-\sqrt{-\Delta} i}+\frac{k+4}{3} \\
& =\omega \sqrt[3]{\|s\| e^{i \theta}}+\omega^{2} \sqrt[3]{\|s\| e^{-i \theta}}+\frac{k+4}{3} \\
& =\frac{\sqrt{k^{2}-k+4}}{3} e^{i \theta / 3} e^{2 i \pi / 3} \\
& +\frac{\sqrt{k^{2}-k+4}}{3} e^{-i \theta / 3} e^{4 i \pi / 3}+\frac{k+4}{3} \\
& =\frac{k+4}{3}+\frac{2 \sqrt{k^{2}-k+4}}{3} \cos \left(\frac{\theta}{3}+\frac{2 \pi}{3}\right),
\end{aligned}
$$




$$
\begin{aligned}
x_{3}= & \omega^{2} \sqrt[3]{-\frac{q}{2}+\sqrt{-\Delta}} i+\omega \sqrt[3]{-\frac{q}{2}-\sqrt{-\Delta}} i+\frac{k+4}{3} \\
= & \omega^{2} \sqrt[3]{\|s\| e^{i \theta}}+\omega \sqrt[3]{\|s\| e^{-i \theta}}+\frac{k+4}{3} \\
= & \frac{\sqrt{k^{2}-k+4}}{3} e^{i \theta / 3} e^{4 i \pi / 3} \\
& +\frac{\sqrt{k^{2}-k+4}}{3} e^{-i \theta / 3} e^{2 i \pi / 3}+\frac{k+4}{3} \\
= & \frac{k+4}{3}+\frac{2 \sqrt{k^{2}-k+4}}{3} \cos \left(\frac{2 \pi}{3}-\frac{2 \theta}{3}\right) .
\end{aligned}
$$

For $0<\theta<\pi$, then $\cos (\theta / 3)>\cos (\theta / 3+2 \pi / 3)$ and $\cos (\theta / 3)>\cos (2 \pi / 3-\theta / 3)$. Thus, $x_{1}>x_{2}, x_{1}>x_{3}$. So the largest root of the univariate cubic equation is

$$
\begin{array}{r}
\mu\left(T_{n, k}\right)=\frac{k+4}{3}+\frac{2 \sqrt{k^{2}-k+4}}{3} \cos \frac{\theta}{3}, \\
\theta=\arccos \frac{2 k^{3}-3 k^{2}-21 k+27 r+11}{2\left(k^{2}-k+4\right)^{3 / 2}}, \\
(0<\theta<\pi) .
\end{array}
$$

For $r=n-1-k$, we have

$$
\begin{array}{r}
\mu\left(T_{n, k}\right)=\frac{k+4}{3}+\frac{2 \sqrt{k^{2}-k+4}}{3} \cos \frac{\theta}{3}, \\
\theta=\arccos \frac{2 k^{3}-3 k^{2}-48 k+27 n-16}{2\left(k^{2}-k+4\right)^{3 / 2}}, \\
(0<\theta<\pi) .
\end{array}
$$

If $k=n-1$, then it is easy to prove that $f(n)=0$. Thus, $\mu\left(T_{n, n-1}\right)=n$.

From Theorems 14, 15, and 21, we have the following corollaries.

Corollary 22. Let $T$ be a tree for $n$ vertices. Then $\mu(T) \leq n$ and equality holds if and only if $T=T_{n, n-1}\left(K_{1, n-1}\right)$, the star with $n$ vertices.

Corollary 23. Let $T$ be a tree for $n$ vertices with $n \geq 4$ and $T \not T_{n, n-1}$. Then

$$
\begin{array}{r}
\mu(T) \leq \frac{n+2}{3}+\frac{2 \sqrt{n^{2}-5 n+10}}{3} \cos \frac{\theta}{3}, \\
\theta=\arccos \frac{2 n^{3}-15 n^{2}+15 n+52}{2\left(n^{2}-5 n+10\right)^{3 / 2}}, \\
(0<\theta<\pi)
\end{array}
$$

and equality holds if and only if $T=T_{n, n-2}(S(1, n-3))$.

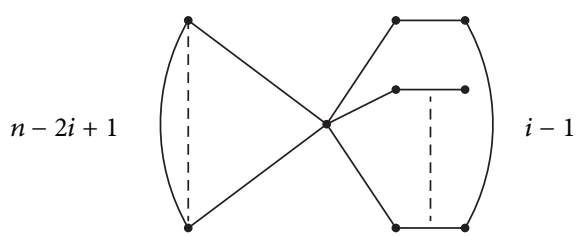

FiguRe 7

Corollary 24. Let $T$ be a tree for $n$ vertices. Let $M(T)$ denote one maximum matching of $T$ and suppose that $|M(T)|=i$. Then

$$
\begin{array}{r}
\mu(T) \leq \frac{n+4-i}{3}+\frac{2 \sqrt{n^{2}-(2 i+1) n+i^{2}+i+4}}{3} \cos \frac{\theta}{3}, \\
\theta=\arccos \frac{2 n^{3}-(6 i+3) n^{2}+\left(6 i^{2}+6 i-21\right) n-2 i^{3}-3 i^{2}+48 i-16}{2\left[n^{2}-(2 i+1) n+i^{2}+i+4\right]^{3 / 2}} \\
(0<\theta<\pi),
\end{array}
$$

and equality holds if and only if $T=T_{n, n-i}$.

Proof. Let $k$ denote the cardinality of the pendant vertices of $T$. For $|M(T)|=i$, we have $k \leq i+n-2 i=n-i$. By Theorems 14 and 15, we have $\mu(T) \leq \mu\left(T_{n, k}\right) \leq \mu\left(T_{n, n-i}\right)$, and equality holds if and only if $T=T_{n, n-i}$.

$T_{n, n-i}$ is shown in Figure 7. Since $i \leq n / 2, n-i \geq n / 2 \geq$ $[n / 2]$, by Theorem 21 we have

$$
\begin{aligned}
& \mu(T) \leq \frac{n+4-i}{3}+\frac{2 \sqrt{n^{2}-(2 i+1) n+i^{2}+i+4}}{3} \cos \frac{\theta}{3}, \\
& \theta=\arccos \frac{2 n^{3}-(6 i+3) n^{2}+\left(6 i^{2}+6 i-21\right) n-2 i^{3}-3 i^{2}+48 i-16}{2\left[n^{2}-(2 i+1) n+i^{2}+i+4\right]^{3 / 2}},
\end{aligned}
$$

$(0<\theta<\pi)$.

Corollary 25. Let $T$ be any tree for $n=2 i$ vertices and $|M(T)|=i$. Then

$$
\begin{array}{r}
\mu(T) \leq \frac{i+4}{3}+\frac{2 \sqrt{i^{2}-i+4}}{3} \cos \frac{\theta}{3}, \\
\theta=\arccos \frac{2 i^{3}-3 i^{2}+6 i-16}{2\left(i^{2}-i+4\right)^{3 / 2}}, \\
(0<\theta<\pi)
\end{array}
$$

and equality holds if and only if $T=T_{n, n-i}$.

\section{Acknowledgments}

The authors would like to thank the anonymous referees for valuable suggestions and corrections, which have improved 
the paper. This research was supported by the Scientific Research Foundation of Huaqiao University (10HZR26) and the Natural Science Foundation of Fujian Province (Z0511028).

\section{References}

[1] F. R. K. Chung, "Eigenvalues of graphs," in Proceedings of the International Congress of Mathematicians, pp. 1333-1342, Zurich, Switzerland, 1995.

[2] F. R. K. Chung, Spectral Graph Theory, American Mathmatical Society, Providence, RI, USA, 1997.

[3] R. Merris, "Laplacian matrices of graphs: a survey," Linear Algebra and its Applications, vol. 197-198, pp. 143-176, 1994.

[4] M. E. Fisher, "On hearing the shape of a drum," Journal of Combinatorial Theory, vol. 1, pp. 105-125, 1966.

[5] S. G. Guo, G. H. Xu, and Y. G. Chen, "The spectral radius of trees with $n$ vertices and diameter $d$," Advances in Mathematics, vol. 34, no. 6, pp. 683-692, 2005.

[6] J.-M. Guo and J.-Y. Shao, "On the spectral radius of trees with fixed diameter," Linear Algebra and its Applications, vol. 413, no. 1, pp. 131-147, 2006.

[7] B. F. Wu, E. L. Xiao, and Y. Hong, "The spectral radius of trees on $k$ pendant vertices," Linear Algebra and its Applications, vol. 395, pp. 343-349, 2005.

[8] Y. Hong and X.-D. Zhang, "Sharp upper and lower bounds for largest eigenvalue of the Laplacian matrices of trees," Discrete Mathematics, vol. 296, no. 2-3, pp. 187-197, 2005.

[9] J.-M. Guo, "On the second largest Laplacian eigenvalue of trees," Linear Algebra and its Applications, vol. 404, pp. 251-261, 2005.

[10] A. Berman and R. J. Plemmons, Nonnegative Matrices in the Mathematical Sciences, Academic Press, New York, NY, USA, 1997.

[11] A. Berman and R. J. Plemmons, Nonnegative Matricesin the Mathematical Sciences, Society for Industrial and Applied Mathematics, Philadelphia, Pa, USA, 1994.

[12] N. Biggs, Algebraic Graph Theory, Cambridge University Press, Cambridge, UK, 2nd edition, 1995.

[13] I. Gutman, D. Vidović, and D. Stevanović, "Chemical applications of the Laplacian spectrum. VI. On the largest Laplacian eigenvalue of alkanes," Journal of the Serbian Chemical Society, vol. 67, no. 6, pp. 407-413, 2002.

[14] B. Mohar, "The Laplacian spectrum of graphs," in Graph Theory, Combinatorics, and Applications, vol. 2, pp. 871-898, 1991.

[15] D. M. Cvetkovic, M. Doob, and H. Sachs, Spectra of Graphs: Theory and Applications, VEB Deutscher Verlag d. Wiss., Berlin, Germany; Academic Press, New York, NY, USA, 1979.

[16] R. Grone and R. Merris, "The Laplacian spectrum of a graph. II," SIAM Journal on Discrete Mathematics, vol. 7, no. 2, pp. 221-229, 1994.

[17] J.-M. Guo, "The effect on the Laplacian spectral radius of a graph by adding or grafting edges," Linear Algebra and its Applications, vol. 413, no. 1, pp. 59-71, 2006.

[18] J.-M. Guo, "A conjecture on the algebraic connectivity of connected graphs with fixed girth," Discrete Mathematics, vol. 308, no. 23, pp. 5702-5711, 2008. 


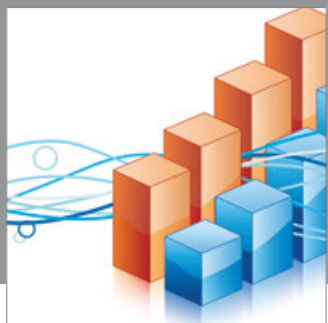

Advances in

Operations Research

mansans

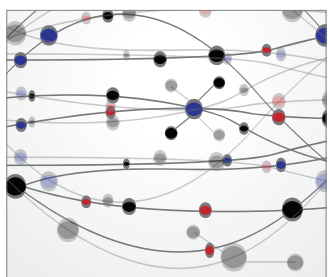

The Scientific World Journal
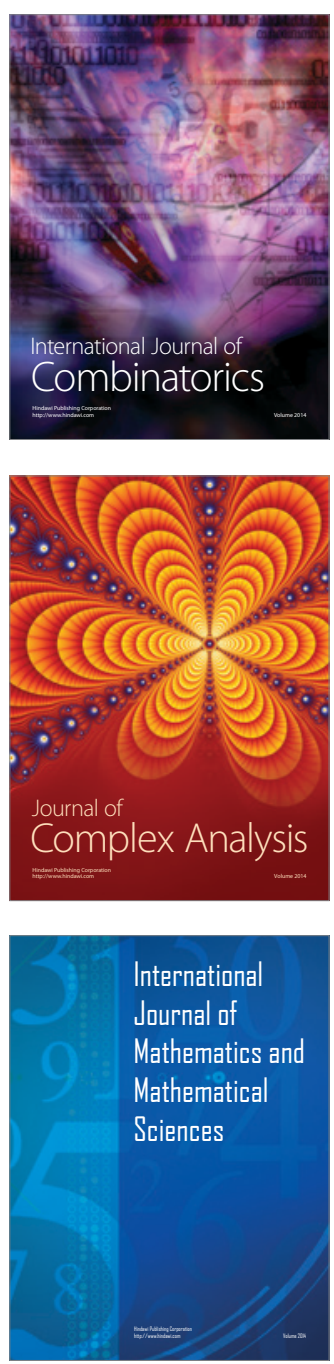
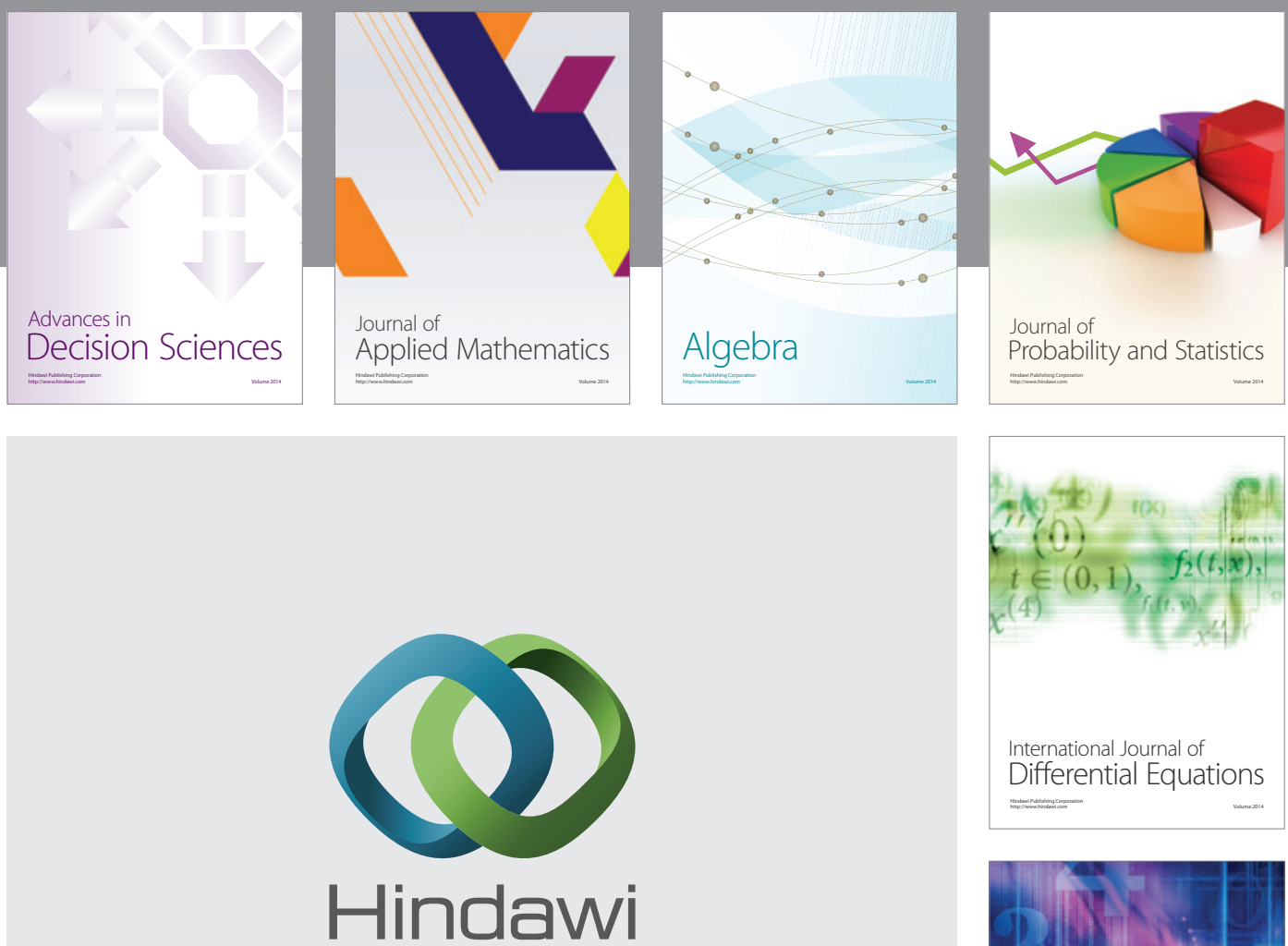

Submit your manuscripts at http://www.hindawi.com
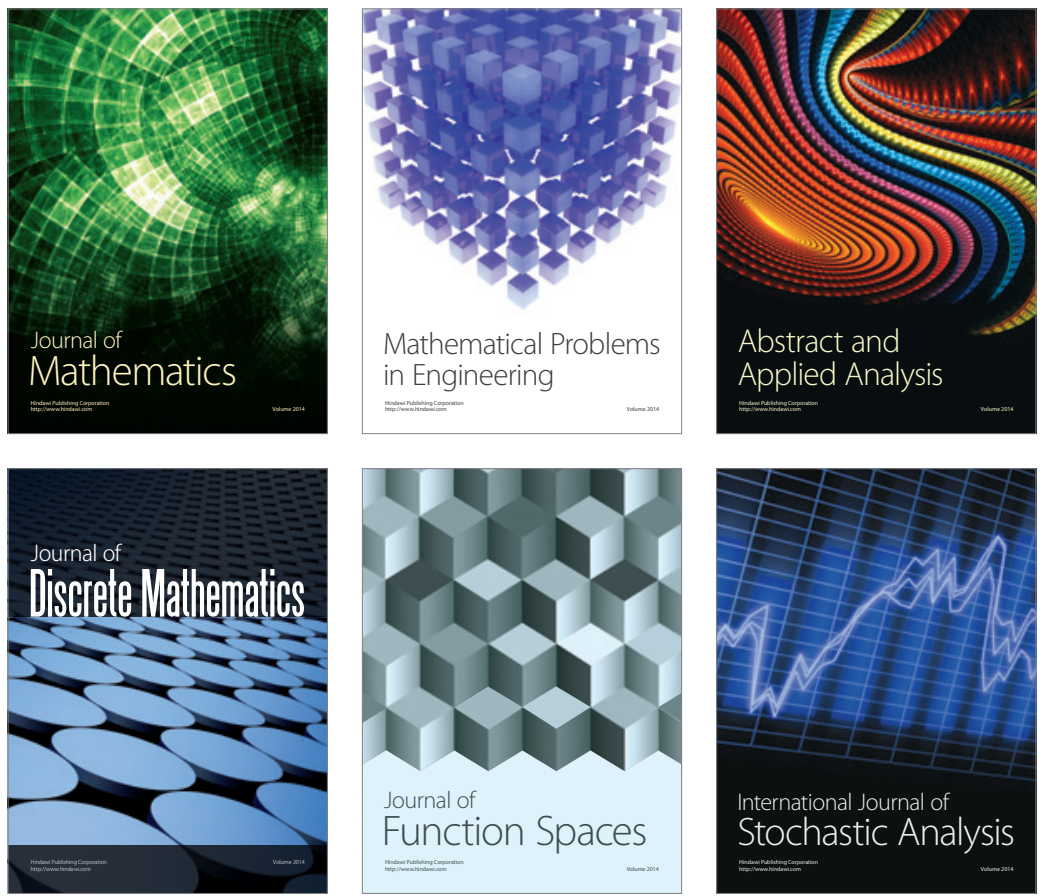

Journal of

Function Spaces

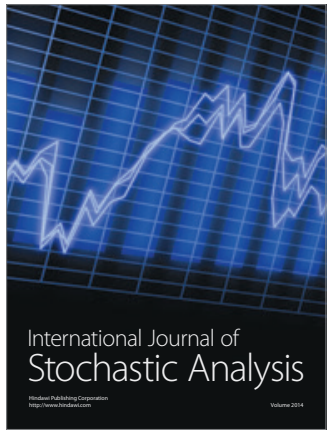

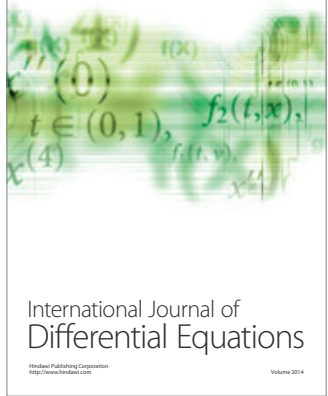
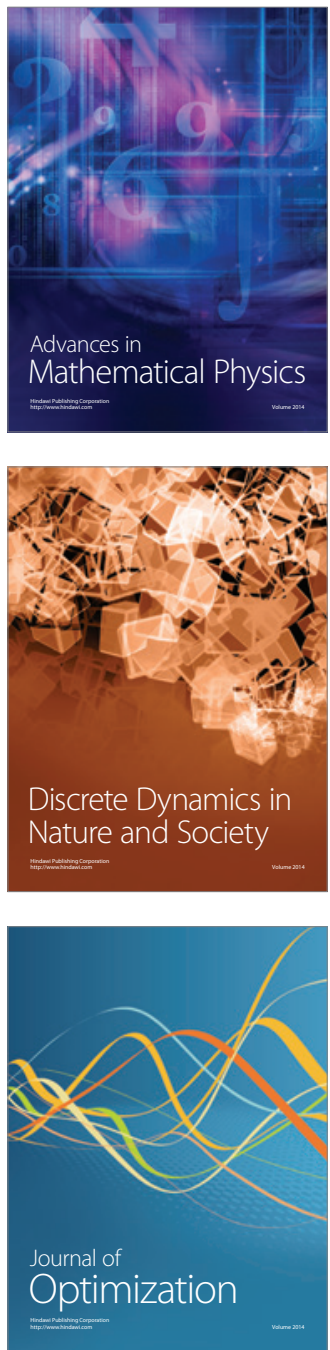